\title{
Melioidosis: clinical aspects
}

\author{
Author: Panduka Karunanayake ${ }^{\mathrm{A}}$
}

Melioidosis is an infectious disease with high mortality and a wide spectrum of clinical manifestations. Successful treatment requires lengthy antibiotic regimens, making microbiological diagnosis crucial. In this article, we briefly review the diagnosis and management of melioidosis from the clinician's viewpoint.

\section{Introduction}

Melioidosis is an infectious disease with high mortality, caused by the bacterium Burkholderia pseudomallei., ${ }^{1,2}$ It is acquired from environmental water or soil. It has a wide spectrum of clinical manifestations, ranging from acute fulminant sepsis to latent infection, and involving almost any organ; it is therefore a great imitator. Most patients have an underlying predisposing medical condition. Successful treatment requires regimens lasting several months to prevent recurrence, making microbiological diagnosis crucial. Timely and aggressive treatment saves lives. Melioidosis can also occur as deadly community outbreaks. In this article, these aspects are briefly reviewed from the clinician's viewpoint.

\section{Epidemiology and pathology}

$B$ pseudomallei is prevalent in South and East Asia and northern Australia within latitudes $20^{\circ} \mathrm{N}$ and $20^{\circ} \mathrm{S}$. But the disease has been reported elsewhere, both as epidemics and sporadic cases, 3,4 and in returning travellers.

It is a gram-negative bacillus, an environmental saprophyte that survives in water and in clay, moist soil for months, and replicates after rain. Humans acquire it through exposure to water or soil, such as during farming, wading or near-drowning in floodwaters, and bathing. It enters the body by inoculation, inhalation or ingestion. Human-to-human transmission has been reported extremely rarely.

It is resistant to components of the innate immune response and can survive inside the lysosomes of neutrophils and macrophages, although interferon- $\gamma$ enhances macrophages' killing capacity. These qualities result in early haematogenous spread. Antibodies are not protective; reduced cell-mediated immunity (eg HIV infection) does not appear to predispose to infection. About threequarters of patients have one or more predisposing conditions $\left(\right.$ Box 1). ${ }^{2}$

Author: ${ }^{\text {A }}$ professor in clinical medicine, University of Colombo, Colombo, Sri Lanka

\section{Box 1. Clinical risk factors for melioidosis for which} good or reasonable evidence is available ${ }^{1}$

$>$ Diabetes mellitus

$>$ Thalassaemia

$>$ Male gender

$>$ Renal disease (renal impairment or failure, calculi)

Excessive alcohol consumption

$>$ Chronic lung disease

Glucocorticoid use

People in endemic areas are exposed to the bacterium from early childhood and become seroconverted; however, clinical disease is uncommon. Disease occurs probably due to two factors: a largerthan-usual inoculum (eg in the rainy season or following neardrowning), and presence of a predisposing condition.

Acute lesions are characterised by acute necrotising inflammation with suppuration, and chronic lesions by inflammatory masses, suppuration, or granulomatous inflammation sometimes with caseation and even giant cells.

\section{Key points}

Melioidosis is a potentially fatal bacterial infection.

The pathogen is a saprophyte in water and soil in tropical and subtropical regions, acquired by inoculation, inhalation or ingestion, especially in the rainy season.

The clinical spectrum is wide, ranging from acute fulminant disease to latent infection with subsequent reactivation. Most patients have an underlying predisposing factor.

Early antibiotic therapy with the correct regimen crucial. It has an intensive phase and an eradication phase.

Management includes supportive care, surgical drainage of abscesses, and control of underlying predisposing factors.

KEYWORDS: melioidosis, Burkholderia pseudomallei, community-acquired sepsis, anti-microbial therapy

DOI: 10.7861/clinmed.2022-0014 


\section{Clinical manifestations}

The clinical spectrum is wide, and it is useful for clinicians to categorise it into four types. ${ }^{5,6}$

The acute septicaemic type occurs after an incubation period of a few days, and presents as a fulminant, community-acquired sepsis that leads to death in a day or two. It is clinically indistinguishable from other similar conditions such as malaria, enteric fever, typhus, leptospirosis, necrotising fasciitis, gram-negative bacteraemia, staphylococcal bacteraemia, invasive pneumococcal disease, invasive meningococcal disease, plague, and Vibrio vulnificus disease. Helpful clues may include seasonal occurrence during rains and epidemiological exposure to water or soil (both of which it shares with leptospirosis), and rapid progression to death and occurrence as an outbreak (both of which it shares with meningococcal sepsis and plague). The pathogen is haematogenously disseminated to lung, liver, spleen, kidney, skin, lymph nodes, subcutaneous tissue, bone, and brain - where small, microscopic abscesses may be detected post-mortem, or where abscesses may be detectable in life if the patient survives long enough. Jaundice, pneumonia, gastroenteritis or meningitis may predominate clinically. The case fatality rate has been as high as $90 \%$ in the past, but can now be reduced to around $12-40 \%$ with early empirical antibiotic therapy and intensive care. ${ }^{1,2}$

The subacute type has a longer and variable incubation period and progresses over weeks to months. The patient presents with milder sepsis and abscesses, often multiple, in lung, liver, spleen, psoas muscle, bone, brain, eyes and soft tissues. If untreated the abscesses may progress to chronic abscesses.

The chronic type may occur de novo or follow the subacute type, either directly or as a recurrence after treatment. The illness can progress slowly and lasts over months to years, and is characterised by chronic abscesses, closely mimicking tuberculosis (even histopathologically), fungal infection or neoplasm.

The latent or asymptomatic type may be detected incidentally in patients who have lived in endemic areas, even years after leaving the area. It can lead to reactivation when the patient's immunity fails, such as following trauma, burns, glucocorticoid administration, cancer, diabetes or other infections such as pneumococcal disease, malaria or influenza A.

In addition, some syndromes are prominent in specific geographical areas: genitourinary infection and prostatic abscess and encephalomyelitis in Australia, and suppurative parotitis in Thailand. ${ }^{1,2}$

\section{Diagnosis}

Non-specific laboratory findings include raised C-reactive proten (CRP) and anaemia. In common with other gram-negative bacteraemias, neutrophil leucocytosis may be absent or slight. Laboratory markers associated with a poor prognosis include leucopenia (especially lymphopenia); a normal or only slightly raised CRP; raised transaminases, bilirubin, urea, and creatinine; hypoglycaemia; and acidosis.

Imaging may point to the diagnosis (eg multiple cavitating consolidations, hepatosplenic abscesses, psoas abscess) and is also invaluable to identify the extent of disease and monitor response to treatment.

The pathogen can be isolated by culture or detected by PCR from a wide range of specimens, including blood, pus, sputum, urine, throat swab and cerebrospinal fluid. It is good practice to take specimens for culture from as many sites as possible, whether clinically abnormal or not, because wide haematogenous dissemination may lead to positivity in any one of them and even one positive culture is diagnostic. ${ }^{2}$

The pathogen grows on conventional culture media, but it is important to communicate with the laboratory staff unless they are familiar with melioidosis, because otherwise the growth may be discarded as a contaminant or 'Pseudomonas'. Specific enrichment and growth media are not available widely.

Serological tests are also available, but they need careful microbiological interpretation.

\section{Treatment}

In the acute septicaemic type, early intensive care for sepsis is crucial. Abscesses should be surgically drained if large enough and accessible. Detection and control of predisposing conditions such as diabetes, kidney disease and immunosuppression are important.

B pseudomallei is intrinsically resistant to the bactericidal antibiotics penicillins (except coamoxiclav), third-generation cephalosporins (except ceftazidime), aminoglycosides and rifamycins. It is relatively resistant to quinolones and macrolides. Among bacteristatic antibiotics, it is sensitive to cotrimoxazole and tetracyclines.

Antibiotic treatment is given in two phases (Box 2). ${ }^{2,7}$ Treatment commences with an intensive phase, to achieve stabilisation, resolution of fever, and a significant improvement of the CRP level. At this point treatment moves to the eradication phase, in order to prevent recurrence of disease.

For the intensive phase, a parenteral (usually bactericidal) antibiotic is used for $2-4$ weeks. Current choice is between ceftazidime and carbapenems (imipenem or meropenem). In the acute septicaemic and subacute types, since microbiological diagnosis is not immediately available and the differential diagnosis is wide, a carbapenem is the frequent choice (often combined with other empirical antibiotics such as vancomycin). In the other types, since antibiotic treatment is often commenced

\section{Box 2. Antibiotics in melioidosis}

Doses may require adjustment in renal failure.

\section{Intensive phase}

One intravenous antibiotic for $2-4$ weeks (longer if necessary), from:

$>$ Ceftazidime $2 \mathrm{~g}$ (70 kg body weight) IV 8-hourly OR

$>$ Meropenem $1 \mathrm{~g}$ ( $2 \mathrm{~g}$ for central nervous system infection) IV 8-hourly OR

Imipenem $1 \mathrm{~g}$ IV 8-hourly

For deep-seated infection (eg central nervous system, prostate, bone, joints), cotrimoxazole 320:1600 mg orally 12-hourly combined with folic acid $5 \mathrm{mg}$ orally daily may be added.

Adjunct therapy (for consideration):

G-CSF $263 \mu \mathrm{g}$ subcutaneously daily for 3 days

\section{Eradication phase}

Two oral antibiotics for 12-20 weeks (longer if necessary), from:

Cotrimoxazole 320:1600 mg orally 12-hourly combined with folic acid 5 mg orally daily

Doxycycline $100 \mathrm{mg}$ orally 12 -hourly

Coamoxiclav 500:125 mg orally 8-hourly 
after microbiological diagnosis, ceftazidime can be the choice; it is combined with oral cotrimoxazole if there are significant deepseated foci not amenable to surgical drainage.

Adjunctive therapy with granulocyte colony-stimulating factor (G-CSF) has been shown to prolong survival to 'buy time' in severe sepsis due to melioidosis, although there was no effect on mortality. ${ }^{8}$

With successful treatment the patient will stabilise and begin to feel better, and the CRP will improve. But the fever may continue for a week or more, and blood cultures may continue to yield the organism. There is no need to reconsider the antibiotic regimen if the patient is clinically improving.

The intensive phase needs to be continued until the patient is afebrile, any abscesses have significantly resolved or been surgically drained, repeat blood cultures are negative, and the CRP is decreased to near-normal levels. This often requires $2-4$ weeks. The time of switching from intensive to eradication phase must be judged carefully, because this is a common time for recurrence.

For the eradication phase, two oral (usually bacteristatic) antibiotics are recommended. Current choice is between cotrimoxazole, doxycycline, coamoxiclav, and chloramphenicol (eg cotrimoxazole plus doxycyline). If monotherapy is considered, coamoxiclav is inappropriate because of a high recurrence rate. Since the eradication phase lasts for months, the side effect profile must be carefully considered in relation to the patient. Although high-dose cotrimoxazole (320:1600 mg 12-hourly) has been recommended, it is not usually well tolerated over prolonged periods.

In survivors, the commonest long-term complication is recurrence of infection. It is important to educate the patient that re-infection is possible, because about half of recurrences are believed to be due to re-exposure and reinfection.

Post-exposure chemoprophylaxis within 48 hours of exposure, with cotrimoxazole or coamoxiclav, is recommended by experts. ${ }^{7}$ Although there is much interest in a large number of non-antibiotic treatments, currently none can be recommended. ${ }^{9}$

\section{Summary}

Melioidosis should be suspected in the appropriate epidemiological setting and clinical picture, and steps taken to confirm the diagnosis microbiologically. In the acute septicaemic and subactue types, early aggressive therapy is essential to save life, and antibiotics must be commenced empirically. Treatment in all four types includes supportive care, surgical drainage of abscesses, and antibiotics. Antibiotic therapy is given as an intensive phase, followed by an eradication phase to prevent recurrence (where the regimen should be tailored to the patient). Since melioidosis can occur as community outbreaks, it is important to inform public health authorities as well.

\section{References}

1 Cheng AC, Currie B]. Melioidosis: epidemiology, pathophysiology, and management. Clin Microbiol Rev 2005;18:383-416.

2 Peacock SJ, Limmathurotsakul D. Melioidosis. In: Cohen J, Powderly WG, Opal SM (eds), Infectious Disease, 4th edn, vol 2. Elsevier, 2017:1073-7.

3 Dance DA. Melioidosis: the tip of the iceberg? Clin Microbiol Rev 1991;4:52-60.

4 Dance DA. Melioidosis as an emerging global problem. Acta Tropic 2000;74:115-9.

5 Acha PN, Szyfres B. Melioidosis. In: Zoonoses and Communicable Diseases Common to Man and Animals, 3rd edn, vol 1: Bacterioses and Mycoses. Pan American Health Organization (Scientific and Technical Publication No. 580), 2006:184-90.

6 Kanai K, Dejsirilert S. Pseudomonas pseudomallei and melioidosis, with special reference to the status in Thailand. Japan J Med Sci Biol 1988:41:123-57.

7 Inglis TJj. The treatment of melioidosis. Pharmaceuticals 2010;3: 1296-303.

8 Cheng AC, Limmathurotsakul D, Chierakul W et al. A randomized controlled trial of granulocyte colony-stimulating factor for the treatment of severe sepsis due to melioidosis in Thailand. Clin Infect Dis 2007:45:308-14.

9 Laws TR, Taylor AW, Russell P, Williamson D. The treatment of melioidosis: is there a role for repurposed drugs? A proposal and review. Expert Rev Anti Infect Ther 2019;17:957-67.

Address for correspondence: Professor Panduka Karunanayake, Department of Clinical Medicine, Faculty of Medicine, 25 Kynsey Road, Colombo 8, 00800, Sri Lanka.

Email: panduka@clinmed.cmb.ac.lk 